\title{
A culturally sensitive model of technology and groups: focus on group support systems
}

\author{
T. L. Griffith \\ University of Arizona and Purdue University, 1310 Krannert Bldg. \\ West Lafayette, IN 47907-1310 USA, 317/494-4485, 317/496-1778 (fax), \\ griffith@mgmt.purdue.edu
}

D. R. Vogel

University of Arizona, McClelland Hall, Tucson, AZ 85721 USA, 520/621-4016,vogel@bpa.arizona.edu

\section{Extended Abstract}

Technological research has arrived at an important nexus. At the same point that research is focusing on the enabling nature of technology in organizations, researchers are also acknowledging issues of culture for management, technology design, and use. The key is that technologies can enable new organizational forms, but the recursive nature of sociotechnical systems is riddled with opportunities for variation in outcomes as these technologies are used (and/or designed) across cultural boundaries. The focus of the current work is to outline a model of sociotechnical system outcomes given differences in culture. A group support system (GSS) context is used to communicate the vividness of cultural variation.

Group Support Systems (GSS) are one example of tools which link technology and groups of organizational participants into a sociotechnical system. In a GSS, users interact with and through computer-mediated communication systems to do collaborative work. Research suggests that GSS can reduce some aspects of process loss inherent in using groups and teams in organizations. Unfortunately, this outcome is predicated on research conducted solely in the U.S. In other cultures, processes losses may occur for different reasons, and/or process "losses" as defined for U.S. groups may not be "losses" in other cultures. The current work expands on these ideas by proposing a broad conceptual model for considering cultural issues in sociotechnical systems.

Definitions for the model's components follow. As noted above, for the purposes of this work technology means GSS. Hofstede suggests that culture is the collective mental programming which distinguishes one group of people from another. Group Process includes the methods of people working together to achieve a common goal. Group Outcomes are the extent to which the group achieves its goals and can include speed, full use of group resources (both task and process), and quality. This is by no means a complete list and would actually be the result of the group's own goals. The indicated links are the result of applying a variety of theories to this context. Connections from one box to another indicate direct effects. Connections from Culture to links 
between other boxes indicate moderating (interaction) effects. Western research has largely provided the support for the links between Technology, Group Process, and Group Outcomes. The links from Culture to Technology and other relationships are where explicit work is lacking.

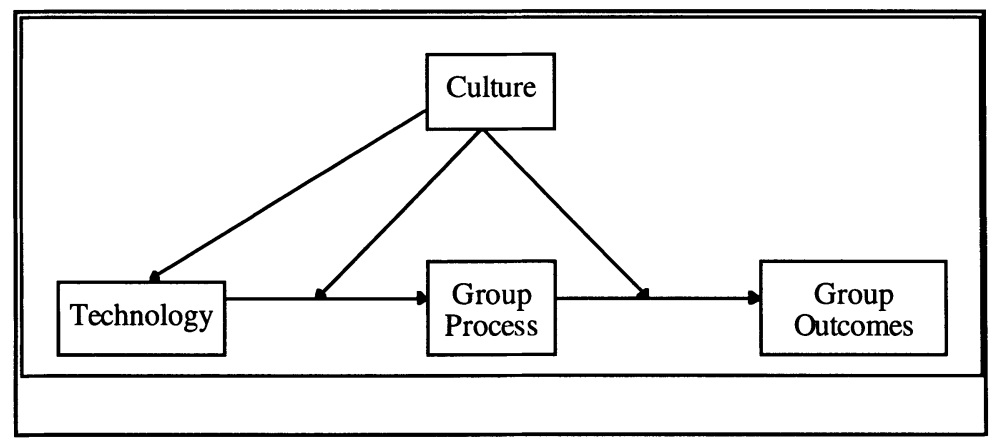

Figure 1 Effects of culture, technology, and group process.

This "white paper" is the beginning of a multi-cultural, longitudinal study using GroupSystems GSS as a focal technology. The long term use of the GSS in a wide variety of settings (GroupSystems is currently in place in over 30 countries) will be studied to consider each of the links in Figure 1. Working from Triandis' cultural dimensions of vertical and horizontal individualism and collectivism, GSS technology can be examined in terms of group process and outcomes. In the Horizontal cultures ( $\mathrm{HC}$ and $\mathrm{HI}$ ), features of GSS that promote equal participation (such as anonymous input) may not be needed. Equal participation is already the norm. However, in Vertical cultures (VC and VI), the GSS features that promote equal participation may have different outcomes depending on whether the culture is Collectivist or Individualist. In VI cultures (such as the US), the structural promotion of equal participation may be necessary and desirable. However, in VC cultures, the promotion of equal participation may have negative outcomes in terms of the disruption of basic social norms and values. VC cultures have learned to work with inequality in a way that maintains relationships. Task oriented technologies that promote information transmission for the betterment of the task may result in more important negative outcomes for the group's relationships.

We propose to use experimental and survey techniques in the examination of the above model. Both technological and group features will be manipulated (e.g., anonymity, status of group members) and measures will include both behavioral and attitudinal outcomes. The results should inform both sides of the sociotechnical model -- designers and implementers will learn the impact of different technology features in different cultural settings, and GSS facilitators will learn how to best utilize both their technological and group process options. 